\title{
On A Hypothetical Mechanism of Interstellar Life Transfer Trough Nomadic Objects
}

\section{Grzegorz Sadlok ${ }^{1}$}

Received: 16 September 2019 / Accepted: 3 January 2020 / Published online: 7 February 2020

(C) The Author(s) 2020

\begin{abstract}
Lethal radiation, low vacuum pressure and low temperatures - this is how space welcomes organisms. Crossing of immense interstellar distances inflates the exposure time of biological material to harmful space conditions. This paper discusses the intriguing possibility of a life-bearing exoplanet being ejected from its planetary system and carrying life across interstellar distances (nomadic $=$ free floating $=$ rogue planet). The proposed interstellar panspermia mechanism reduces the exposure time to space conditions and provides multiple chances for interactions between microbes-bearing rock debris and exoplanets within system the nomadic object encountered on its way. The testing strategy is outlined and discussed in the paper, including testable predictions the proposed hypothesis makes.
\end{abstract}

Keywords Panspermia · Ejected exoplanets · Super Earths · Jovian moons · Interstellar transit

\section{Introduction}

Panspermia is an old concept (see Arrhenius 1908; Kamminga 1982; Kawaguchi 2019) there are at least a few versions of the idea. All differ in a mode the life is transferred from one exoplanet to another. Some proposals include even actions of intelligent beings - the so called directed panspermia (see e.g. Arrhenius 1908; Crick and Orgel 1973; Burchell 2004; Nicholson 2009). The working model of panspermia is that life can leave the home planet (via natural processes), travel through space, survive the journey and colonize a new world.

The mechanism could permit life to be a common phenomenon in the Universe, no matter how easy or difficult the abiogenesis would be. Once life had formed somewhere, it could spread everywhere. However, there is an obvious drawback to testing this concept - the only

\section{Grzegorz Sadlok}

gregsadlok@gmail.com

1 Faculty of Natural Sciences, Institute of Earth Sciences, University of Silesia in Katowice, Będzińska 60 Street, PL-41-200 Sosnowiec, Poland 
life we know so far is the one on Earth. Despite this shortage of data, some progress has been made regarding theoretical modes of transportation available and strategies microbes could use to deal with the space environment (e.g. Nicholson et al. 2005; Nicholson 2009).

Cosmic space is not a welcoming environment. Life in open space is exposed to lethal radiation, high vacuum and low temperatures. A feasible solution to deal with some of the challenges is lithopanspermia - a hypothetical process according to which mineral matter (rock, sediment, dust) protects biological payload from radiation of space (Horneck 1993). Lithopanspermia postulates that microbes may leave the home planet/moon during impact events along with rock bits being ejected into space (see Horneck et al. 2001). Those microbebearing rock fragments become later projectiles hitting and fertilizing the surfaces of other worlds (Nicholson 2009, and references therein). Such a mechanism of life transfer seems to be most feasible between exoplanets of tight systems (Fig. 1a), e.g. around M-dwarfs where transit and space-exposure times are relatively short (e.g. Lingam and Loeb 2017). The total mass of rock fragments ejected from a planet would increase with the size of impactor - larger impacts would generate more debris. Beech et al. (2018) estimates that large events, like K/T impact could generate as much as $5.5 \times 10^{12} \mathrm{~kg}$ of debris with individual boulders being $1.8 \mathrm{~m}$ across and $8 \times 10^{3} \mathrm{~kg}$ in weight.

Higher frequency of impact events would also generate more ejected rock fragments. Therefore, lithopanspermia would likely work more effectively in younger planetary systems with more rock and ice fragments floating around and increasing the overall chances of impact events. It is interesting that the oldest known traces of life on Earth (isotopic signatures) show up in rocks formed c.a. 3.8 Ga ago (Mojzsis et al. 1996) - apparently life appeared on Earth's surface shortly after the late heavy bombardment had ended (Gomes et al. 2005; Michel and Morbidelli 2007).

\section{Interstellar Panspermia}

Panspermia on an interstellar scale however is a different story. Microbes crossing the interstellar distances would have to deal with the unfriendly space environment over thousands and millions of years required for completion of the journey across light years separating planetary systems.

Melosh (2003) showed that it is unlikely that any rock bit originating on a terrestrial planet within our Solar System could ever hit similar terrestrial planet of another planetary system in this view, the lithopanspermia would be confided to exchange within the Solar System. On the contrary, Napier (2004) argued that meter-scale boulders ejected from Earth are destroyed through collisions with other objects and with dust particles in space. After collision remnants reach a size of a micron, the radiation pressure overcomes the gravity and those tiny particles with their bio-payloads are ejected from the system with a rate of $10^{20}$ per million years (see Napier 2004). Wallis and Wickramasinghe (2004) looked at direct transfer of ejecta - from a planetary surface to another world in a different planetary system - and at indirect route employing comets. They concluded that both processes are ineffective unless protoplanetary discs of stellar nebulas are considered. A similar concept was pursued by Adams and Spergel (2005) who analyzed the chances of life exchange between stars in star forming clusters where planetary systems are closer to each other and move at lower relative speeds. Zubrin (2019) showed recently that interaction between Oort Clouds of approaching star systems may play a role in life exchange at the interstellar scale - the approaching stars may disturb the Oort Clouds of each other and capture foreign comets. An exchange of planets between systems 


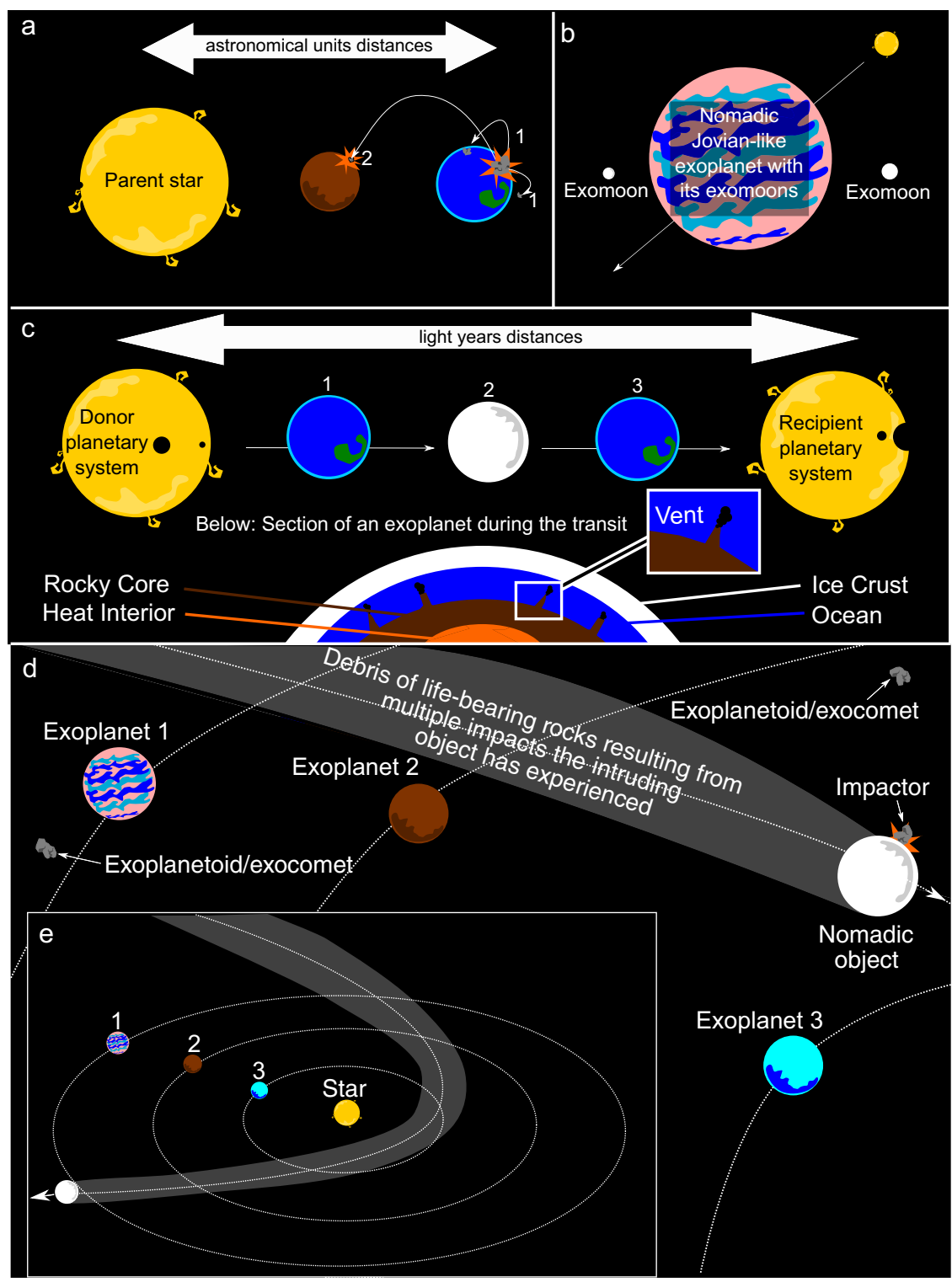

Fig. 1 Schematic illustration explaining the proposed hypothesis of interstellar panspermia. a Lithopanspermia an impact ejects rock material with microbes (transfer at scale of astronomical units). Larger fragments fall back on the planet (1), smaller may travel to a nearby planet within the same system (2). b Jupiter-mass planetary objects are common among ejected nomadic exoplanets. Presence of Jovian-style exomoon systems would open an interesting option for interstellar life transfer, tidal forces could keep some of those moons habitable during the transit. c Mechanism of interstellar (light years scale) life transfer via a nomadic exoplanet: life-bearing object gets ejected from its system (1) and covered with ice during the transit (2). Internal heat is required to keep the under-ice liquid ocean; exoplanet approaches another system - interstellar transit is completed (3). At this stage processes of traditional lithopanspermia may allow for surface-to-surface life transfer. $\mathbf{d}$ An intruding object crosses orbits of a few exoplanets (1, 2 and 3). The intruder gets hit multiple times with space-floating rock fragments. It leaves a trail of debris, including its own, life-bearing rock fragments. Each exoplanet whose orbit has been crossed by the intruding object may interact twice a year with the debris trail. Exoplanets having shorter orbital periods and those having stronger g-fields have greater chances of successful life transfer 
during their fly-bys is also a possible scenario in young star clusters (see Malmberg et al. 2011) and could potentially serve as a mechanism of interstellar life exchange.

Recently, the concept of interstellar material exchange found a support in first observations of extrasolar objects (an asteroid and a comet) entering our system (see Fitzsimmons et al. 2018; Hallatt and Wiegert 2019). Such interstellar material exchange could be a potential mechanism allowing the transfers of biological material between planetary systems. However, the amount of rock and ice material being exchanged between star systems may be limited (e.g. Wallis and Wickramasinghe 2004; Adams and Spergel 2005; Zubrin 2019) and the chances of rock bits being captured by a nearby planetary system are slim (Wallis and Wickramasinghe 2004; Adams and Spergel 2005). Hence, to render such a mechanism effective, microbes would have to be common in rock bits floating in space - just to ensure that they will be present in those few fragments being exchanged between the systems. As yet, unequivocal microbes or their fossils have not been found in meteorites which fell on Earth, including those meteorites that could originate on Mars (see Kerr 1997). It appears to be a logical conclusion that microbe-bearing rock fragments must be rare in space. Therefore, as a result the rock/ice material exchange may be rather an ineffective life transfer mechanism at interstellar scale (see Melosh 2003).

The solution to a successful interstellar panspermia may be not to leave the home exoplanet for interstellar voyage at all, but rather to take it along (Fig. 1). Several authors have suggested that nomadic objects floating in space could harbor life (see Stevenson 1999; Abbot and Switzer 2011; Durand-Manterola 2010; Badescu 2010, 2011a, 2011b; Strigari et al. 2012) and that such objects could play a role in the interstellar panspermia (Durand-Manterola 2010; Abbot and Switzer 2011; Strigari et al. 2012). Durand-Manterola (2010) offered most details and proposed a mechanism in which the atmosphere of intruding nomadic object would be stripped off by the stellar wind. The microbes released this way in space would be dragged by the wind and could colonize the planets of the invaded system. However, this proposal ignores the UV sensitivity of the microbes. Durand-Manterola (2010) mentions also that the collisions with the intruder could have played a role in life transfer through formation of life-bearing fragments. However, he provides no further details on this mechanism. The aim of this paper is to take a closer look at this intriguing proposal and to offer a hypothetical testable mechanism for interstellar panspermia via nomadic objects.

\section{Hypothesis}

A life-bearing exoplanet is ejected from its unstable planetary system and becomes a planetaryscale nomadic object (Fig. 1c). The object travels the interstellar space and approaches other planetary system(s). It enters the new system and gets hit with that system's ice/rock debris planetoids and comets, bits left over from that system's accretion. The impacts eject microbebearing fragments of the intruder into space. The rock bits form a trail of debris along the object's path (Fig. 1d). The fragments stay in orbit around the star for some time and the trail serves as a repository of life-bearing rock fragments. Exoplanets whose orbits have been cut by the intruder may interact with the debris trail twice a year (Fig. 1e).

The interstellar transit time will depend on relative velocity of the nomadic object and the approached system and on a distance to that system. However, the transit time is a negligible factor in the proposed mechanism. This is because nomadic objects should be able to sustain heat and life for periods of time at scale of $10^{9}$ years (see Stevenson 1999, Abbot and Switzer 
2011) - larger terrestrial exoplanets would cool down slower than the smaller ones. Therefore, such nomadic objects should be able to sustain their biospheres for a longer time than smaller ones. If the intruding object is too large however, e.g. a Jovian-class planet, it may have a disruptive gravitational effect on the approached system, leading to destabilization of orbits. Finally, the proposed mechanism would work best for intruders approaching the system in ecliptic plane or close to it. This is because most of the rock and ice debris left after system's accretion is expected to rotate approximately in this plane and thus the frequency of impacts would be the greatest for objects with trajectories close to the ecliptic plane.

Plausible candidate objects to carry life across interstellar distances are Earth or Super-Earth class terrestrial nomadic exoplanets or Jovian-like exomoons associated with large Jupiter-size nomadic exoplanets (see Sasselov and Valencia 2010; Lammer et al. 2009; Heller and Armstrong 2014; Hong et al. 2018). The gravitational influence of the Jupiter-mass exoplanet may render such exomoons habitable during the interstellar journey (see Heller and Armstrong 2014).

The proposed types of celestial bodies are likely to have shielding rocks, $\mathrm{H}_{2} \mathrm{O}$ (water/ice) and internal heat source. Water is a key component for habitability (see Kasting et al. 1993, Stevenson 1999). Massive, Earth-like exoplanets should create their own internal heat. Smaller objects, e.g. exomoons of a Jupiter-class exoplanet, could generate heat due to tidal forces they experience within the g-field of exoplanets they orbit.

Without an atmospheric protection the $\mathrm{H}_{2} \mathrm{O}$ would be mostly frozen during the interstellar transit but the heat source would provide volcanic activity - volcanoes or vents could warm up the lower parts of the $\mathrm{H}_{2} \mathrm{O}$ cover, keeping some of it in a liquid state: the ice cover with water ocean underneath (see Abbot and Switzer 2011; Fig. 1c herein). Such a model nomadic object would resemble Europa, one of Jupiter's moons (see Carr et al. 1998, and references therein). Abbot and Switzer (2011) showed that thinner ice cover is required to keep under ice liquid water for a less massive nomadic planet if the planet has more water than Earth and/or if its surface is covered with a thick $\mathrm{CO}_{2}$ layer.

Stevenson (1999) proposed a category of plausible life-bearing unbound objects with atmospheres. He argued that ejected planets may keep their original nebula gas envelopes composed of $\mathrm{H}_{2}$ - low temperature leads to high pressure at the base of such an atmosphere ( $10^{2}$ to $\left.10^{4} \mathrm{bar}\right)$. The pressure causes far-infrared opacity of the $\mathrm{H}_{2}$. Therefore such nomadic objects could be cold $(\sim 30 \mathrm{~K})$ but still they could have oceans of water at their surfaces - oceans with temperatures and pressures comparable to those at the ocean-floors of Earth (Stevenson 1999). Badescu (2010, 2011a, 2011b) reached similar conclusions modeling various composition of optically thick gas envelopes of unbound planets. Therefore, such planets having optically thick or opaque atmospheres are also plausible life-bearing nomadic objects for interstellar transfer mechanism.

\section{Founding Data}

\section{Nomadic Objects}

Exoplanets may be ejected from their parent planetary systems due to various processes (e.g. Rasio and Ford 1996; Weidenschilling and Marzari 1996; Malhotra 2002; Barnes and Quinn 2004; Malmberg et al. 2011; Veras and Raymond 2012; Bakari et al. 2018). Such expelled objects become nomadic and travel through interstellar space without a star. Such unbound objects are also known in the literature as rogue planets and free-floating planets. Theoretical estimations showed there may be potentially $10^{5}$ unbounded objects per-main-sequence star in 
the Galaxy, such objects could range in mass from $10^{-8}$ to $10^{-2}$ of Sun's masses (see Strigari et al. 2012). The unbounded planets may intrude into encountered foreign planetary system(s). Such meetings may be just a fly-by or they may end in a capturing of an intruder by the encountered system (see Goulinski and Ribak 2017).

Recent gravitational microlensing studies indicate that Jupiter-mass exoplanets may occur with frequency up to 0.25 exoplanet per main-sequence star (compare Sumi et al. 2011; Quanz et al. 2012; Mróz et al. 2017). The existence of such massive, unbound planetary objects has been already confirmed by direct observations with near-infrared imaging (see Zapatero Osorio et al. 2000) and with microlensing (see Mróz et al. 2018, 2019). Earth-mass and Super-Earth-mass exoplanets are detectable with ultrashort microlensing events and recent preliminary studies suggest they may be common in space (see Mróz et al. 2017).

\section{Frequencies of Earth-Mass and Super-Earth-Mass Exoplanets}

Kepler's data suggest that exoplanets similar in size to Earth (0.5 to 2 Earth's radius) may occur with frequency of 0.5 exoplanet per red dwarf (Kopparapu 2013). Petigura et al. (2013) found, extrapolating Kepler's data, that $\sim 5.7 \%$ of Sun-type stars may harbor an exoplanet comparable in size and orbital period to Earth.

\section{Earth's Light-Independent Biota}

The planetary ejection would likely terminate light-dependent biota. However, simple, lightindependent ecosystems could survive. Biological processes of chemotrophic and heterotrophic microorganisms would likely operate as usual during the interstellar transit, as long as chemical components and heat would be provided from the object's interior. There are such light-independent ecosystems on Earth, one can find them at hydrothermal vents at the sea floors (see Cann et al. 1997; Flores and Reysenbach 2011; Abbot and Switzer, 2011). Interestingly, hydrothermal vents ecosystems may have been among the first that emerged on a young Earth (see Dodd et al. 2017).

\section{Testing Strategy}

Panspermia may be confirmed only if common ancestry is proven for biological entities from two different celestial objects. Therefore, scientific the testing of panspermia concept is a major challenge. Ultimately, it will be possible only with physical evidence at hand - acquisition of extraterrestrial biological material. Testing of interplanetary panspermia, for example in EarthMars system (e.g. Mileikowsky et al. 2000), will be possible as soon as our understanding improves of the Martian surface. This may happen only after manned exploration will have started (years from now?). However, testing of interstellar panspermia will have to await much longer (centuries from now?) as travel between stars is still beyond our technological capabilities regarding both, manned and unmanned missions (see Dyson 1968).

\section{Evaluation of the Hypothesis Components}

The preliminary evaluation of the hypothesis will require addressing the following questions with new observatory data: 
1. How frequent are Earth-mass and Super-Earth-mass nomadic objects?

2. How frequent is $\mathrm{H}_{2} \mathrm{O}$ on exoplanets orbiting other stars?

3. Does volcanic activity depend on size of terrestrial exoplanets?

4. Do Jovian moons have habitable oceans under their ice-covers?

Improved microlensing detection should answer the question on how frequent are nomadic Earth-mass and Super-Earth-mass planetary objects (see e.g. Strigari et al. 2012; Mróz et al. 2017; Bennett et al. 2018; Penny et al. 2019; Hamolli et al. 2019; question 1). Improved understanding on composition of atmospheres of rocky exoplanets (their spectral analysis) will shed light on how frequent $\mathrm{H}_{2} \mathrm{O}$ is (see e.g. Ballesteros et al. 2019; question 2). Atmospheric composition (e.g. $\mathrm{CO}_{2}$ abundance) may give also a hint of information on volcanic activity as $\mathrm{CO}_{2}$ is one of its typical products (see Giggenbach 1996); the $\mathrm{CO}_{2}$ presence is important in models of Abbot and Switzer (2011) where an under-ice water ocean may be sustained despite the small mass of the planet only if a $\mathrm{CO}_{2}$ layer is present at the planet's surface. Therefore, volcanic activity $\left(\mathrm{CO}_{2}\right.$ abundance $)$ of planets smaller than Earth may improve their ability to sustain liquid water under the ice and life during the transit (question 3). Future mission(s) to Europa moon will help to understand how a $\mathrm{H}_{2} \mathrm{O}$-rich world with internal heat source may behave away from a star providing the heat - including conditions under the ice (question 4).

\section{Predictions Made by the Hypothesis}

The proposed panspermia scenario permits some testable predictions to be made regarding the life-bearing exoplanets.

The rock debris trail left by intruding life-bearing nomadic object could serve for some time as a repository of life-bearing rock bits. Its presence would provide multiple opportunities life transfer windows - for fertilizing exoplanets within the system. Exoplanets having shorter orbital periods would interact with the debris trail more frequently than those with longer periods. Also, the time during which the biological material in space-floating rock bits could stay fertile would be likely limited by microbes' abilities to stay in a dormant state (see Seaward et al. 1976; Vreeland et al. 2000). Therefore, the overall higher frequency of exoplanet-debris interaction would increase the chances of successful life transfer.

The strength of planetary g-filed would determine how effective an exoplanet is at draining the rock bits from the debris trail. Exoplanets having stronger g-fields would drain the trail debris more effectively - more bits would end ultimately at their surfaces.

Therefore, the proposed scenario predicts that the overall chances for successful fertilization increase with

- decreasing exoplanet's orbital period;

- increasing exoplanet's g-field.

The mentioned predictions need to co-occur with life-suitable conditions on the planet receiving the life-bearing fragments in order to plant the life successfully (e.g. planet in a habitable zone). The case of Earth matches the proposed model. The planet had a suitable environment (habitable zone), it has a short orbital period and the greatest g-field of all the inner planets of the Solar System. However, not all planetary systems are like our system and they may differ significantly in configurations. For example, planetary systems with gas giants - large Jupiter-size planets - orbiting close to their host stars ("hot Jupiter"; see e.g. Henry 
et al. 2000) may be less welcoming for life transferred via the proposed mechanism. The giant planet will drain most of the rock bits from the debris trail left by the intruder. In such a scenario life would likely not survive on a hot gas giant. However, if the planet had a Jovianlike moon system and some of those moons had conditions suitable for life then there would be a chance of successful fertilization of the moons.

If the proposed mechanism of panspermia is valid then life should be more common on exoplanets matching the tight orbit and strong g-field criteria. These predictions should be testable if and when life-signatures are remotely detected through spectral analysis of exoplanets' atmospheres. A database with a population of potential life-harboring exoplanets will be required to test the proposed predictions.

\section{Conclusions}

The proposed hypothesis postulates that life can transit interstellar distances on nomadic exoplanets and/or its exomoons.

Erath-mass, Super-Earth-mass exoplanets and Jovian-like exomoons are proposed as carrier-objects. During the interstellar transit such words would resemble Europa, Jupiter's moon, having an ice crust and a subsurface liquid water ocean. Also, exoplanets and exomoons with thick infrared-opaque atmospheres could sustain life during the transit.

The current state of knowledge supports the hypothesis and shows Jupiter-size nomadic exoplanets are frequent. Preliminary observations show Earth-mass and Super-Earth-mass objects may be common as well.

The scenario limits the time exposure of biological material to lethal radiation, low vacuum pressure and low temperatures during the interstellar transit in open space (see DurandManterola 2010). Light-independent ecosystems (similar to those around Earth's hydrothermal vents) could survive the ejection from planetary system and interstellar voyage.

The nomadic object entering new planetary system would disturb system's planetoids and comets, it could receive multiple impacts and would likely form along its path a trail of debris including its own life-bearing rock bits. Such a debris trail could serve as a repository of lifebearing rock fragments. Exoplanets within the system could interact with the trail twice a year. Those having shorter orbital periods and those with stronger planetary g-fields would have higher chances of successful fertilization. Therefore, life transferred in the way outlined in the proposed scenario should be more frequent on exoplanets having shorter orbital periods and stronger g-fields.

Acknowledgments I am grateful to an anonymous reviewer of Origins of Life and Evolution of Biospheres. His/her comments significantly improved the manuscript. I am grateful to Alan W. Schwartz (Radboud University, Netherlands), the Editor-in-Chief of Origins of Life and Evolution of Biospheres, for his valuable comments and linguistic corrections.

Open Access This article is licensed under a Creative Commons Attribution 4.0 International License, which permits use, sharing, adaptation, distribution and reproduction in any medium or format, as long as you give appropriate credit to the original author(s) and the source, provide a link to the Creative Commons licence, and indicate if changes were made. The images or other third party material in this article are included in the article's Creative Commons licence, unless indicated otherwise in a credit line to the material. If material is not included in the article's Creative Commons licence and your intended use is not permitted by statutory regulation or exceeds the permitted use, you will need to obtain permission directly from the copyright holder. To view a copy of this licence, visit http://creativecommons.org/licenses/by/4.0/. 


\section{References}

Abbot DS, Switzer ER (2011) The Steppenwolf: a proposal for a habitable planet in interstellar space. Astrophys J 735:L27

Adams FC, Spergel DN (2005) Lithopanspermia in star-forming clusters. Astrobiology 5:497-514

Arrhenius S (1908) The transmission of life through the universe. Monist 18:161-175

Badescu V (2010) Tables of Rosseland mean opacities for candidate atmospheres of life hosting free-floating planets. Open Physics 8:463-479

Badescu V (2011a) Constraints on the free-floating planets supporting aqueous life. Acta Astronautica 69:788-808

Badescu V (2011b) Free-floating planets as potential seats for aqueous and non-aqueous life. Icarus. 216:485-491

Bakari A, Haghighipour N, Do A (2018) On the origin of free floating planets: planet ejection during terrestrial planet formation. AAS/division for planetary sciences meeting abstracts 50: id. 113.13

Ballesteros F, Fernandez-Soto A, Martínez V (2019) Diving into exoplanets: are water seas the Most common? Astrobiology 19:642-654

Barnes R, Quinn T (2004) The (in) stability of planetary systems. Astrophys J 611:494-516

Beech M, Coulson IM, Comte M (2018) Lithopanspermia-the terrestrial input during the past 550 million years. American Journal of Astronomy and Astrophysics 6:81-90

Bennett DP, Akeson R, Anderson J, Armus L, Bachelet E, Bailey, V et al (2018) The WFIRST exoplanet microlensing survey. arXiv preprint: arXiv:1803.08564

Burchell MJ (2004) Panspermia today. Int J Astrobiol 3:73-80

Cann JR, Elderfield H, Laughton A, Juniper SK, Tunnicliffe V (1997) Crustal accretion and the hot vent ecosystem. Philosophical transactions of the Royal Society of London. Series a: mathematical. Phys Eng Sci 355:459-474

Carr MH, Belton MJS, Chapman CR, Davies ME, Geissler P, Greenberg R et al (1998) Evidence for a subsurface ocean on Europa. Nature 391:363-365

Crick F, Orgel L (1973) Directed panspermia. Icarus 19:341-346

Dodd MS, Papineau D, Grenne T, Slack JF, Rittner M, Pirajno F et al (2017) Evidence for early life in Earth's oldest hydrothermal vent precipitates. Nature 543:60-64

Durand-Manterola HJ (2010) Free-floating planets: a viable option for panspermia. arXiv prerpint: arXiv: 1010.2735

Dyson FJ (1968) Interstellar transport. Phys Today 21:41-45

Fitzsimmons A, Snodgrass C, Rozitis B, Yang B, Hyland M, Seccull T et al (2018) Spectroscopy and thermal modelling of the first interstellar object 1I/2017 U1 'Oumuamua. Nature Astronomy 2:133-137

Flores GE, Reysenbach AL (2011) Hydrothermal environments, marine. In: Reitner J, Volker T (eds) Encyclopedia of Geobiology. Springer Netherlands, Dordrecht, pp 456-467

Giggenbach WF (1996) Chemical composition of volcanic gases. In: Scarpa R, Tilling RI (eds) Monitoring and mitigation of volcano hazards. Springer, Berlin Heidelberg, pp 221-256

Gomes R, Levison HF, Tsiganis K, Morbidelli A (2005) Origin of the cataclysmic late heavy bombardment period of the terrestrial planets. Nature 435:466-469

Goulinski N, Ribak E (2017) The capture rate of free-floating planets in our galaxy. EPSC Abstracts: European Planetary Science Congress 11:1-2

Hallatt T, Wiegert P (2019) The dynamics of interstellar asteroids and comets within the galaxy: an assessment of local candidate source regions for 1I/ Oumuamua and 2I/Borisov. arXiv preprint: arXiv:1911.02473

Hamolli L, Hafizi M, Paolis FD, Nucita AA (2019) Free-floating planets in the milky way Arabian journal of mathematics https://doi.org/10.1007/s40065-019-0267-3: 1-9

Heller R, Armstrong J (2014) Superhabitable worlds. Astrobiology 14:50-66

Henry GW, Marcy GW, Butler RP, Vogt SS (2000) A Transiting “51 Peg-like” Planet. Astrophys J 529:L41-L44

Hong YC, Raymond SN, Nicholson PD, Lunine JI (2018) Innocent Bystanders: Orbital Dynamics of Exomoons During Planet-Planet Scattering 852:85

Horneck G (1993) Responses of Bacillus subtilis spores to space environment: results from experiments in space. Orig Life Evol Biosph 23:37-52

Horneck G, Stöffler D, Eschweiler U, Hornemann U (2001) Bacterial spores survive simulated meteorite impact. Icarus 149:285-290

Kamminga H (1982) Life from space - a history of panspermia. Vistas Astron 26:67-86

Kasting JF, Whitmire DP, Reynolds RT (1993) Habitable zones around main sequence stars. Icarus 101:108-128

Kawaguchi Y (2019) Panspermia hypothesis: history of a hypothesis and a review of the past, present, and future planned missions to test this hypothesis. In: Akihiko Y, Takeshi K, Tomohiro U (eds) Astrobiology: from the origins of life to the search for extraterrestrial intelligence. Springer, Singapore, pp 419-428

Kerr RA (1997) Putative Martian microbes called microscopy artifacts. Science 278:1706-1707 
Kopparapu RK (2013) A revised estimate of the occurrence rate of terrestrial planets in the habitable zones around Kepler M-dwarfs. The Astrophysical Journal Letters 767:1-5

Lammer H, Bredehöft JH, Coustenis A, Khodachenko ML, Kaltenegger L, Grasset O et al (2009) What makes a planet habitable? The Astronomy and Astrophysics Review 17:181-249

Lingam M, Loeb A (2017) Enhanced interplanetary panspermia in the TRAPPIST-1 system. Proc Natl Acad Sci 114:6689-6693

Malhotra RA (2002) Dynamical mechanism for establishing apsidal resonance. Astrophys J 575:L33-L36

Malmberg D, Davies MB, Heggie DC (2011) The effects of fly-bys on planetary systems. Mon Not R Astron Soc 411:859-877

Melosh HJ (2003) Exchange of meteorites (and life?) between stellar systems. Astrobiology 3:207-215

Michel P, Morbidelli A (2007) Review of the population of impactors and the impact cratering rate in the inner solar system. Meteorit Planet Sci 42:1861-1869

Mileikowsky C, Cucinotta FA, Wilson JW, Gladman B, Horneck G, Lindegren L et al (2000) Natural transfer of viable microbes in space: 1. From Mars to Earth and Earth to Mars. Icarus 145:391-427

Mojzsis SJ, Arrhenius G, McKeegan K, Harrison T, Nutman A, Friend C (1996) Evidence for life on earth before 3,800 million years ago. Nature 384:55-59

Mróz P, Udalski A, Skowron J, Poleski R, Kozłowski S, Szymański MK et al (2017) No large population of unbound or wide-orbit Jupiter-mass planets. Nature 548:183-186

Mróz P, Ryu YH, Skowron J, Udalski A, Gould A, Szymański MK (2018) A Neptune-mass free-floating planet candidate discovered by microlensing surveys. Astron J 155(121):1-6

Mróz P, Udalski A, Bennett DP, Ryu YH, Sumi T, Shvartzvald Y (2019) Two new free-floating or wide-orbit planets from microlensing. Astronomy \& Astrophysics 622(A201):1-8

Napier WMA (2004) A mechanism for interstellar panspermia. Mon Not R Astron Soc 348:46-51

Nicholson WL (2009) Ancient micronauts: interplanetary transport of microbes by cosmic impacts. Trends Microbiol 17:243-250

Nicholson WL, Schuerger AC, Setlow P (2005) The solar UV environment and bacterial spore UV resistance: considerations for earth-to-Mars transport by natural processes and human spaceflight. Mutation Research/ Fundamental and Molecular Mechanisms of Mutagenesis 571:249-264

Penny MT, Bachelet E, Johnson S, Beaulieu JP, Kerins E, Rhodes J et al (2019) Measurement of the free-floating planet mass function with simultaneous Euclid and WFIRST microlensing parallax observations. arXiv preprint: arXiv: 1903.08180

Petigura EA, Howard AW, Marcy GW (2013) Prevalence of earth-size planets orbiting sun-like stars. Proc Natl Acad Sci 110:19273-19278

Quanz SP, Lafreniere D, Meyer MR, Reggiani MM, Buenzli E (2012) Direct imaging constraints on planet populations detected by microlensing. Astronomy \& Astrophysics 541(A133):1-4

Rasio FA, Ford EB (1996) Dynamical instabilities and the formation of extrasolar planetary systems. Science 274:954-956

Sasselov DD, Valencia D (2010) Planets we could call home. Sci Am 303:38-45

Seaward M, Cross T, Unsworth BA (1976) Viable bacterial spores recovered from an archaeological excavation. Nature 261:407-408

Stevenson DJ (1999) Life-sustaining planets in interstellar space? Nature 400:32

Strigari LE, Barnabè M, Marshall PJ, Blandford RD (2012) Nomads of the galaxy. Mon Not R Astron Soc 423: 1856-1865

Sumi T, Kamiya K, Bennett D, Bond I, Abe F, Botzler C et al (2011) Unbound or distant planetary mass population detected by gravitational microlensing. Nature 473:349-352

Veras D, Raymond SN (2012) Planet-planet scattering alone cannot explain the free-floating planet population. Mon Not R Astron Soc Lett 421:L117-L121

Vreeland RH, Rosenzweig WD, Powers DW (2000) Isolation of a 250 million-year-old halotolerant bacterium from a primary salt crystal. Nature 407:897-900

Wallis MK, Wickramasinghe NC (2004) Interstellar transfer of planetary microbiota. Mon Not R Astron Soc 348: 52-61

Weidenschilling SJ, Marzari F (1996) Gravitational scattering as a possible origin for giant planets at small stellar distances. Nature 384:619-621

Zapatero Osorio MR, Béjar VJS, Martın EL, Rebolo R, Navascués DB, Bailer-Jones CAL, Mundt R (2000) Discovery of young, isolated planetary mass objects in the $\sigma$ orionis star cluster. Science 290:103-107

Zubrin R (2019) Exchange of material between solar systems by random stellar encounters. International journal of astrobiology https://doi.org/10.1017/S1473550419000144: 1-6

Publisher's Note Springer Nature remains neutral with regard to jurisdictional claims in published maps and institutional affiliations. 